\title{
Manufacturing and assembly status of main components of the Wendelstein 7-X Cryostat
}

\author{
Torsten Koppe $^{1)}$, Dr. A. Cardella ${ }^{1)}$, J. Reich ${ }^{1)}$, B. \\ Missal $^{1)}$, B. Hein ${ }^{1)}$, R. Krause ${ }^{1)}$, H. Jenzsch ${ }^{1)}$, D. \\ Hermann $^{1)}$, Dr. M. Schrader ${ }^{1)}$, \\ ${ }^{1)}$ Max-Planck-IPP, EURATOM Assoc., \\ D-17491 Greifswald Germany \\ torsten.koppe@ipp.mpg.de
}

\author{
G. Di Bartolo ${ }^{2)}$, F. Leher ${ }^{3)}$, A. Binni ${ }^{3)}$, J. Segl ${ }^{3)}$, R. \\ Camin $^{4)}$, Dr. L. Giordano ${ }^{5)}$, S. Langone ${ }^{6}$, Prof. Dr. J. \\ Ridzewski $^{7)}$, Giorgio Corniani ${ }^{8)}$ \\ ${ }^{2)} \mathrm{M} \& \mathrm{G}$ srl Consultants, Giussano, Italy \\ ${ }^{3)}$ MAN DWE GmbH, AB - Plant Construction, \\ Deggendorf, Germany \\ 4) Equipos Nucleares, S.A., Maliano, Spain \\ ${ }^{5)}$ CLP, Borgo san Dalmazzo, Italy \\ ${ }^{6)}$ Romabau Gerinox AG, Weinfelden, Schweiz \\ ${ }^{7)}$ IMA GmbH, Dresden Germany \\ ${ }^{8)}$ Ettore Zanon s.p.a., I-36015 Schio (Vi), Italy
}

\section{INTRODUCTION}

Abstract -Wendelstein 7-X (W7-X) will be the world's largest superconducting helical advanced stellarator. This stellarator fusion experiment is at present in assembly at the Max-PlanckInstitut für Plasmaphysik (IPP). W7-X is deemed to be a desirable alternative for a future power plant like DEMO. The main advance of the static plasma is caused by the threedimensional shape of some of the main mechanical components inside the Cryostat.

The toroidal plasma with a ring diameter of $11 \mathrm{~m}$ and an average plasma diameter of $1.1 \mathrm{~m}$ is contained within the Plasma Vessel. Its form is dictated by the shape of the plasma. Its geometry is formed around the three-dimensional shape of the plasma. The form of the plasma is controlled by the coil system configuration. In order to control the plasma form it is necessary that all the $\mathbf{2 0}$ planar and 50 non planar coils be positioned within a tolerance of $1.5 \mathrm{~mm}$. To meet this requirement a complex coil support structure was created, consisting of the Central Support Ring and the different inter coil supports. The coils and the support structure are enclosed within the Outer Vessel with its domes and openings. The space between the Outer and the Plasma Vessel is called Cryostat because the vacuum inside provides thermal insulation of the magnet system. The entire magnetic system is then cooled down to $4 \mathrm{~K}$. Due to different thermal movements the Plasma Vessel and the Central Support Ring have to be supported separately. The Central Support Ring is held by 10 Cryo Legs. The Plasma Vessel supporting system is divided into two separate systems, allowing horizontal and vertical adjustments to centre the Plasma Vessel during thermal expansion.

This paper aims to give an overview of the main components in the Cryostat like the Plasma Vessel, the Outer Vessel, the ports and the different support systems. It describes the current manufacturing and assembly status and the associated problems of these components, using pictures and text.

Wendelstein 7-X, Stellerator, Cryostat, Plasma Vessel, Outer Vessel, Magnetic System, Coil Support Structure, Central Support Ring, Cryo Legs
W7-X is the world's largest superconducting helical advanced stellarator (Fig. 1). It is presently under construction at the Max-Planck-Institut für Plasmaphysik in Greifswald, Germany. W7-X is provided with an internal vacuum vessel, the 'Plasma Vessel', whose complex toroidal shape follows the designed contour of the stellarator plasma. The Plasma Vessel is the first barrier for the ultra-high vacuum $\left(\leq 10^{-8} \mathrm{mbar}\right)$ of the plasma chamber. The superconducting magnet system, the coil support structure, the liquid helium cooling pipes and the thermal shield are enclosed between the Plasma Vessel and a second external toroidal vacuum vessel, the 'Outer Vessel'. An intermediate vacuum $\left(\leq 10^{-6} \mathrm{mbar}\right)$ is established between the vessels

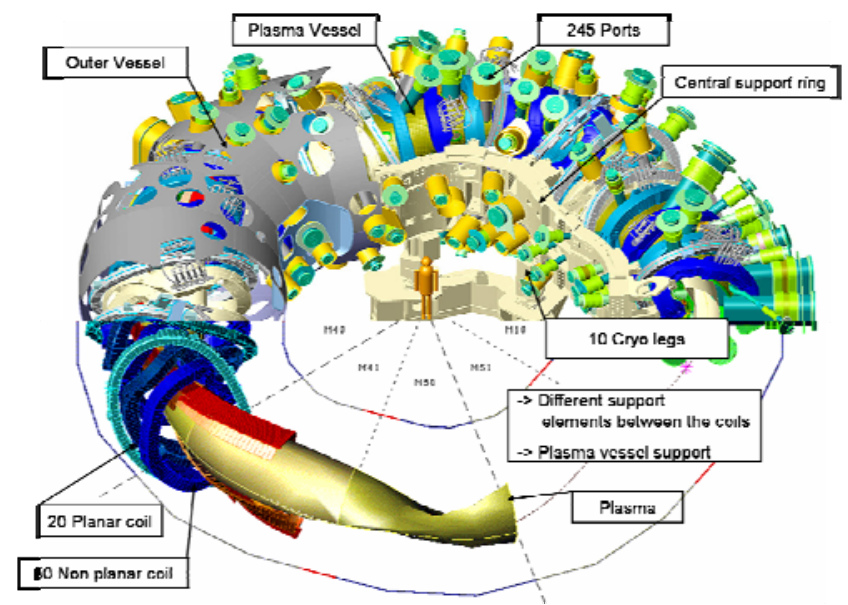

Figure 1. Main Cryostat components of Wendelstein 7-X 


\section{Plasma Vessel}

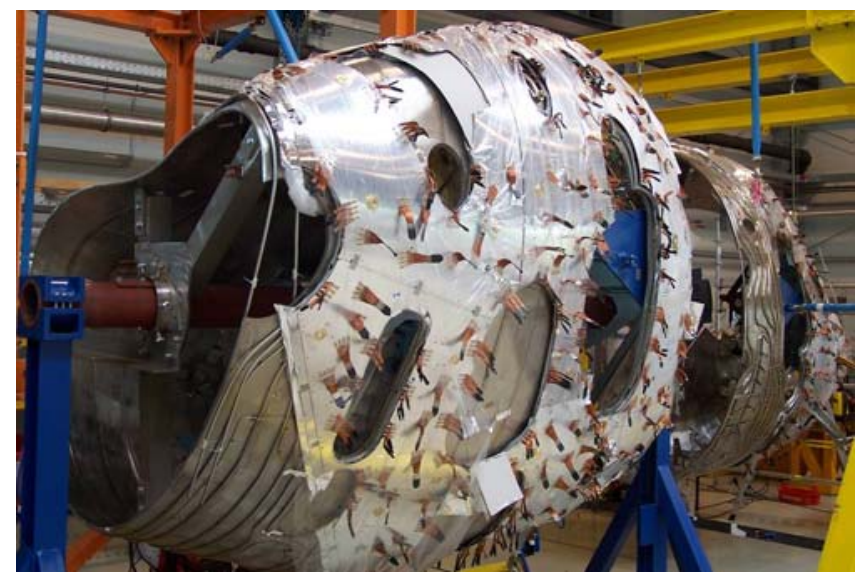

Figure 2. Plasma Vessel of module 5 of Wendelstein 7-X

The Plasma Vessel consists of 5 similar modules, each extending for $72^{\circ}$ in a toroidal direction, which are joined together along radial-poloidal and radial-toroidal planes. The inner surface is protected against the plasma heat by the actively cooled panels of the in-vessel components. Its outer wall is covered with the thermal shield consisting of an actively cooled glass fibre and copper shell with multi-layer super insulation which minimises the thermal radiation from the Plasma Vessel to the coils [1].

The maximum outer diameter of the helical twisted Plasma Vessel torus is approximately $12 \mathrm{~m}$; the minimum inner diameter is $8 \mathrm{~m}$. The Plasma Vessel is made of the austenitic steel 1.4429 (X2CrNiMoN17-13-3) and has a wall thickness of $17 \mathrm{~mm}$. The openings for the 254 ports are distributed evenly around the Plasma Vessel torus. The shape of the Plasma Vessel cross-section changes within each module from a triangular to a bean form and back again to a triangular form. Because of this complicated form, surfaces switch between convex and concave.

For the manufacturing the Plasma Vessel was divided in further smaller parts and supports. Each module consists of two half modules which are joint together by two single sections. The supplier MAN DWE GmbH (Deggendorf, Germany) manufactured and delivered the Plasma Vessel parts and connected components. The assembly of the thermal isolation and the welding on the assembly stands is on the internal time schedule. Also all Vertical Plasma Vessel Supports were delivered by MAN DWE GmbH

\section{OUTER VESSEL}

The Outer Vessel and the Plasma vessel form the boundaries for the Cryostat. The Outer Vessel is designed as a torus with an outer diameter of approximately $16 \mathrm{~m}$. The internal diameter of the cross section is $4.4 \mathrm{~m}$. It is made of austenitic steel 1.4429, the same material as the Plasma Vessel. The nominal wall thickness of the shell is $25 \mathrm{~mm}$. The Outer Vessel is also made of 5 modules. Unlike the Plasma Vessel each Outer Vessel module is divided into an upper and a lower half-module shell. The Outer Vessel rests on 15 supports, which are directly connected to the machine foundation. The access between the outer and the inner vessel is achieved via ports and domes. 524 domes were welded onto the torus [2].

The Outer Vessel serves as the outer vacuum protection for all cold components inside the Cryostat. The thermal insulation of the magnet system is provided by a high vacuum in combination with the super isolation and the active cooled thermal radiation shield. Because the surface of the Outer Vessel is at ambient temperature, it will be covered by the super isolation shield inside the Cryostat.

All modules of the Outer Vessel were manufactured. At present the first module is insulated with the thermal isolation. The manufacturer is also MAN DWE GmbH.

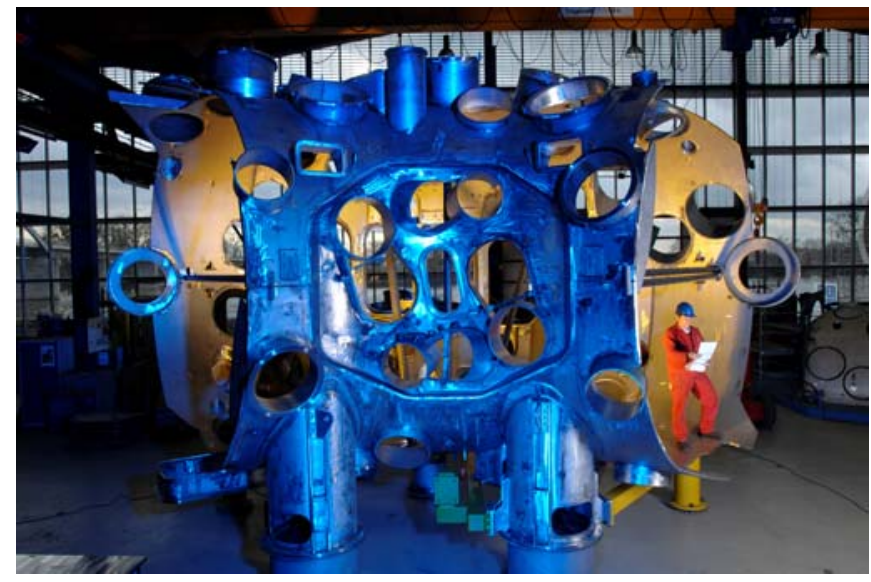

Figure 3. One module of the Outer Vessel during a test assembly

\section{PORTS}

The 254 ports provide the access to the Plasma Vessel. All ports are equipped with bellows which vary between $100 \mathrm{~mm}$ circular to $1170 \times 570 \mathrm{~mm}$ rectangular sizes. The bellows allow relative movements between the Outer and the Plasma Vessel. During various load-cases the axial and lateral stiffness of all bellows will create a resulting spring-force which acts directly on the vessel supports.

The ports can be divided in two main groups. The so-called diagnostic ports contain the different systems for observing the plasma. The supply ports are used for the heating systems (i.e. ECRH, ICRH and NBI), the supply lines of in-vessel components and maintenance. All ports were delivered to the Greifswald site. The ports were built by the Swiss company Romabau-Gerinox AG, Weinfelden.

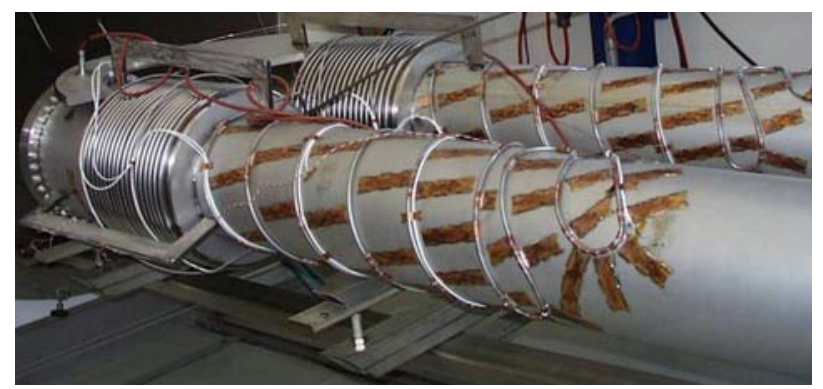


Figure 4. Diagnostic ports of Wendelstein 7-X during a leak test

\section{SUPPORT STRUCTURE OF THE MAGNETIC SYSTEM}

The Cryostat encloses different support systems for the magnetic system. The so-called Central Support Structure (CSS) consists of several types of structural components. The main one is the Central Support Ring to which the superconducting coils are connected through Central Support Elements (CSE).

The CSS is subjected to various loads during the life of the W7-X experiment. The main loads are generated during coil de-energisation and subsequent magnetic forces. Additional loads can occur due to cooling down and the dead weight of the CSS. All of these loads were taken into account in the structural analysis of the CSS.

\section{A. Central Support Ring}

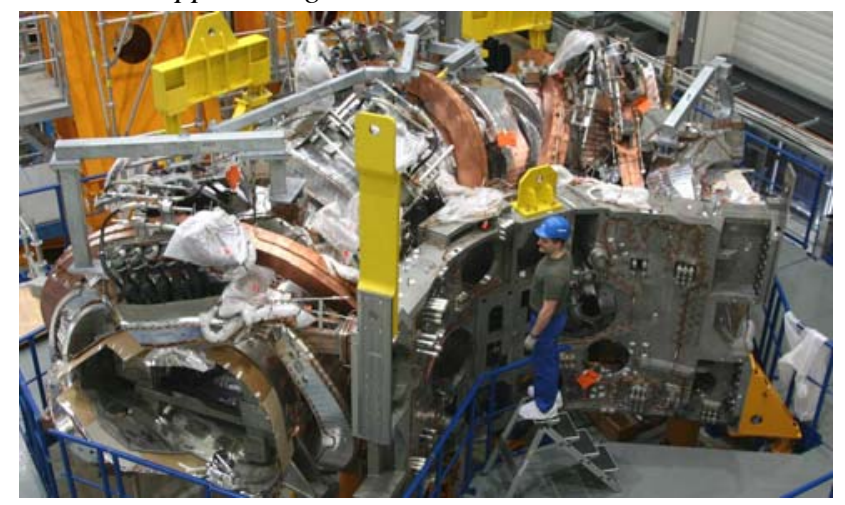

Figure 5. Central Support Ring half module of W7-X at the assembly stand

The magnetic system consisting of 20 planar and 50 non planar coils is supported by a pentagonal $10 \mathrm{~m}$ diameter, $2.5 \mathrm{~m}$ high coil support structure. This structure is called Central Support Ring (CSR) and consists of a mix of 1.3960 and 1.4429 steel plates welded together. Also the CSR is divided into five modules. Each module is split in two equal half modules. The ring has two main flange interfaces that connect the individual CSR half-modules. Both ring parts are connected by a step flange at the half module interface. The half module flange is bolted by standard bolts and some shear bolts to avoid movements during operation. At the module interface a flat flange configuration is used to connect the modules. Due to the risk of a flange face misalignment after the positioning of neighbouring models, shear bolts can not be used with the module flange. Instead shim plates and wedges will achieve the best fit possible [3].

Four of the five CSR modules were completed and delivered to IPP at the beginning. Three were successfully assembled with the coils meeting the tight manufacturing tolerances. The fourth half module was also delivered to IPP. The final machining of the last module was successfully completed. The ten half modules of the CSR are built by the Spanish company Equipos Nucleares SA, Maliano. The mechanical machining is carried out by the Italian company CLP (Borgo san Dalmazzo, Italy).

\section{B. Inter Coil Supports}

In order to resist the high magnetic forces during operation the coils support each other by a number of different support elements. 60 Lateral Support Elements (LSE) connect the coils on the outside of the magnetic system. LSE interconnect the non planar coil on the outboard side and can transfer both forces and moments in all directions. The LSE add toroidal stiffness to the torus and thus limit deflections at the far end of the non planar coil. The 245 Narrow Support Elements (NSE) will endure the high magnetic forces on the inner side and at the smallest gaps [4]. Unlike the LSE they allow both a movement and a tilting of the coils. The 80 Planar Support Elements (PSE) are the link between the planar and non planar coils. Due to the higher flexibility of the planar coils the PSE were introduced to limit the deflection during operation. There are two main types of PSE a rigid bolted connection and a contact support. The 20 Contact Elements (CE) are placed between the coils at the half module and module junction [5].

For the first three modules all Inter Coil Supports were delivered and assembled except the Inter Coil Supports at the module junction. At this time the fourth module is in assembly. The required Inter Coil Supports are pre-manufactured. After further measurements during the assembling process the final machining will be done.

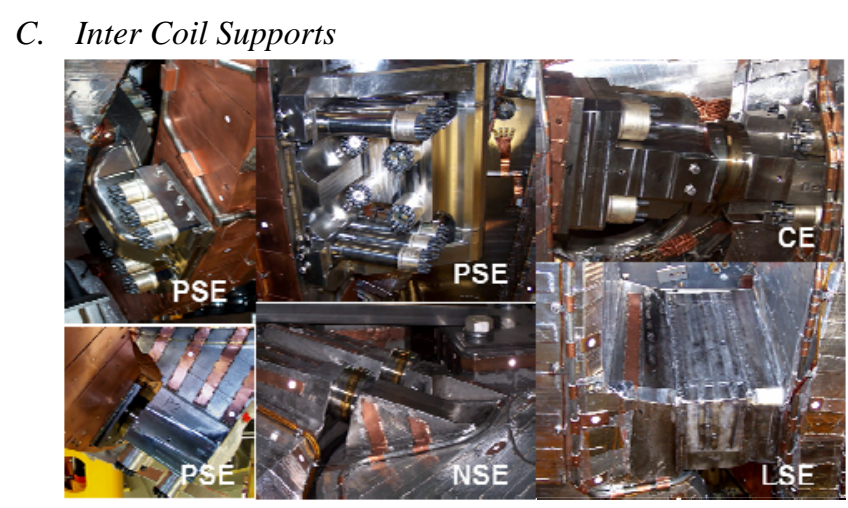

Figure 6. Different inter coil supports of Wendelstein 7-X

In order to resist the high magnetic forces during operation the coils support each other by a number of different support elements. 60 Lateral Support Elements (LSE) connect the coils on the outside of the magnetic system. LSE interconnect the non planar coil on the outboard side and can transfer both forces and moments in all directions. The LSE add toroidal stiffness to the torus and thus limit deflections at the far end of the non planar coil. The 245 Narrow Support Elements (NSE) will endure the high magnetic forces on the inner side and at the smallest gaps [4]. Unlike the LSE they allow both a movement and a tilting of the coils. The 80 Planar Support Elements (PSE) are the link between the planar and non planar coils. Due to the higher flexibility of the planar coils the PSE were introduced to limit the deflection during operation. There are two main types of PSE a rigid bolted connection and a contact support. The 20 Contact Elements (CE) are placed between the coils at the half module and module junction [5]. 
For the first three modules all Inter Coil Supports were delivered and assembled except the Inter Coil Supports at the module junction. At this time the fourth module is in assembly. The required Inter Coil Supports are pre-manufactured. After further measurements during the assembling process the final machining will be done.

\section{Connection Parts}

All connection elements for the coils and their support structure are listed as Connection Parts. The Connection Parts can not be identified as one single component. They consist of tensioners, shims, back plates and wedges. Connection Parts are bolting connections where Superbolts ${ }^{\circledR}$ are used into the threaded holes on the connection blocks on the coils. Tightening the bolts presses the flange faces from the ring and coils together to form a frictional joint. Shoulders and wedges around the flange faces provide additional support against large shear and bending loads coming from the coils. In addition special Connection Parts are used for the different connections of the CSR and Inter Coil Supports.

For the first three modules all Connection Parts were delivered and assembled except the Connection Parts at the module junction. At this time the fourth module is in assembly. The required Connection Parts were delivered.

\section{E. Cryo Leg}

The CSR with the connected coils has to be supported by the machine base. Each module is held by two Cryo Legs on the bottom side of the CSR. Due to the fact that the Cryo Leg is partially located within the Cryostat and partially outside, it also has to ensure proper insulation to prevent heat transfer into the Cryostat. This is achieved by introducing a glass fibre reinforced plastic (GRP) pipe between the steel sections of the Cryo Leg. The Cryo Leg has to be able to adapt the movements caused by the cooling down of the magnetic system. This cooling down will introduce radial shrinkage towards the machine centre in the order of $15 \mathrm{~mm}$ at the radius of the Cryo Leg location. This shrinkage is allowed by the Cryo Leg through the use of sliding bearings between Cryo Leg and machine base. In addition a callot bearing is used to eliminate transfer of moments to the Cryo Leg base. The Cryo Leg is loaded with the deadweight of the CSS. In addition the Cryo Leg sees loads during operation as a result of CSR deformation, which again is caused by the de-energised coils. The glass fibre reinforced plastic pipe is fitted into a stainless steel ring on the outer ends of the tube [6].

All Cryo Legs were delivered by Ettore Zanon (Schio, Italy). The glass fibre reinforced plastic pipes were produced and thoroughly checked by IMA GmbH (Dresden, Germany).

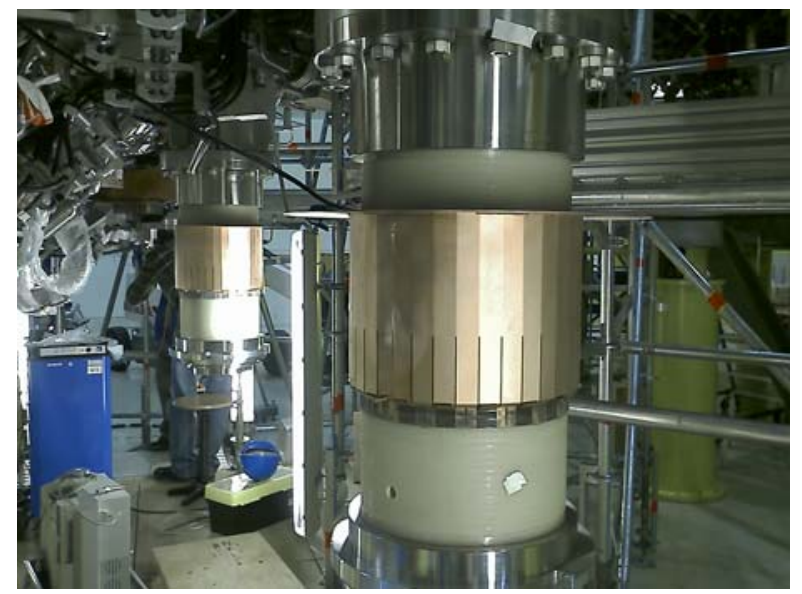

Figure 7. Cryo Leg of Wendelstein 7-X

\section{CONCLUSION}

The assembly of four of the five modules is progressing well. Most of the main parts in the Cryostat were not only delivered but also assembled. They could show that the assembly requirements were achieved. In general it can be resumed that all parts which are named in this paper are on the recent internal time schedule. From this point of view there are no doubts to complete the assembly up to 2014. But because of the complexity of the parts and the tight tolerances the production process poses a great challenge to the contractors. Nevertheless the manufacturing of the main Cryostat components is progressing well and most of the requirements were met.

\section{REFERENCES}

[1] J. Reich, Manufacture of Cryostat components for Wendelstein 7-X, Fusion Eng. Des. 69 (2003) 345-348

[2] A. Cardella, Construction of the Vacuum Vessels and the Magnet Supporting, Structures of WENDELSTEIN 7-X, proc. 24th SOFT, Warshaw 11-15/9/2006.

[3] F. Schauer, Status of Wendelstein 7-X Construction, proc. 24th SOFT, Warshaw 11-15/9/2006

[4] J. Reich, Manufacture of Inter-Coil Support-Elements of the W7-X Magnet System, Sofe 2007

[5] R. Krause, Spezifikation NSE Halbzeuge, Technical Specification, 1AAA00HH5 S-0000.1, IPP documentation, 28.07.2006

[6] H. Jenzsch, Test von LSE Dummies, Specification, 1-AAA00HH0 S0000.0, IPP documentation, 10.02.2006 